Check for updates

Cite this: RSC Adv., 2018, 8, 20968

\title{
Preparation and sound absorption properties of barium titanate/nitrile butadiene rubber- polyurethane foam composites with stratified structure
}

\author{
Xueliang Jiang, ${ }^{\text {ab }}$ Zhijie Wang, ${ }^{\text {ab }}$ Zhen Yang, ${ }^{\text {ab }}$ Fuqing Zhang, ${ }^{\text {ab }}$ Feng You ${ }^{\text {ab }}$ \\ and Chu Yao (iD *ab
}

Barium titanate/nitrile butadiene rubber (BT/NBR) and polyurethane (PU) foam were combined to prepare sound-absorbing materials with different stratified structures including a double-layer structure and alternating multilayered structure, respectively. The effects of the cell size of the PU foam and the thickness of the PU foam layer on the sound absorption efficiency of the BT/NBR-PU foam composite with a double-layer structure were studied, and the effects of the alternating unit number on the sound absorption efficiency of the BT/NBR-PU foam composite with an alternating multilayered structure were studied. The results show that the sound absorption peak of the double-layer structure composites would move toward low frequency with a decrease of the cell size of the PU foam or with an increase the thickness of the PU foam layer. With increasing alternating unit number, the composites with an alternating multilayered structure have good sound absorption performance in a wider frequency bandwidth. The sound absorption frequency range of the stratified composite could be adjusted by changing the cell size of the PU foam, the thickness of the PU foam layer and the alternating unit number. Each stratified structure BT/NBR-PU foam, whether with a double-layer structure or alternating multilayered structure, shows excellent sound absorption efficiency at low frequency owing to the combination of airflow resistivity, resonance absorption and interface dissipation.

Received 18th April 2018

Accepted 1st June 2018

DOI: $10.1039 / \mathrm{c} 8 \mathrm{ra03330g}$

rsc.li/rsc-advances efficiency at low frequency was poor if the material merely had single structure and sound absorption mechanism. The main reason is that the low frequency sound waves attenuate slowly in air, could navigate around obstacles easily, and has strong penetrability compared with medium and high frequency sound waves. Therefore, low frequency sound waves are hard to be absorbed. Moreover, low frequency noise is more long-term harm to human health than high frequency noise and will result in hearing impairment, neurasthenia and cardiovascular system hazards. ${ }^{4-6}$ The poor sound absorption performance at low frequency has become an important factor which restricts the development of sound-absorbing material. Nowadays, it is the main idea to improve efficiency of low-frequency sound absorption that several kinds of structures and sound absorption mechanisms are combined in the same sound-absorbing composite material. Because of having damping effect and low density, the sound absorbing composite with polymer matrix receives many attentions and is considered having a promising prospect.

The selection of polymer matrix, the functionality of filler and the design of structure are regarded as the key factors to improve the low-frequency sound absorption performance of the sound absorbing composite with polymer matrix. Many 
kinds of rubber and plastic matrix polymers with high damping and low density, as the matrix, were combined with multiple functional fillers such as ramie fiber, ${ }^{7}$ carbon nanotubes, ${ }^{8}$ piezoelectric ceramics, ${ }^{9}$ and so on. After a great deal of work, it was found that the increase of the low frequency sound absorption efficiency could not be achieved by altering the matrix or the fillers simply. Hence, many researches were conducted in structure design as well, and various structures were investigated such as porous structure, ${ }^{10,11}$ periodic structure, ${ }^{12,13}$ helical structure,${ }^{14}$ multilayer structure,${ }^{15}$ corrugated sandwich structure,$^{16}$ perforated structure, ${ }^{17,18}$ and so on. Yang ${ }^{19}$ reported a periodic unit cell model and compared the effects of the fullyopen cells and the semi-open cells on the sound absorption performance. $\mathrm{Liu}^{20}$ put forth the localized resonant theory of sonic crystals and provided the theory basis for application of sonic crystals in low-frequency sound-absorbing field. However much of the studies on the sound-absorbing materials with sonic crystals structure is still theoretical. $\mathrm{Xue}^{21}$ reported a microporous poly(vinyl formal) foams prepared by a two-step acetalization method, and found that the structure could absorb the sound waves effectively. Zhao ${ }^{15}$ reported an foam/ film poly(ethylene-co-octene) composite with alternating multilayer structure, and found that the composite had good sound absorption performance at medium and high frequency. In summary, to some extent the sound absorption efficiencies of materials were increase through the above meaningful work. However, these sound absorption materials did not show excellent behavior at low frequency. Therefore, further investigations are needed about how the design of structures and the combination of sound absorption mechanisms could become preferable and more reasonable.

A lot of work has been done and previously reported by the author on the preparation of organic-inorganic composites and the structure design of sound absorbing materials. ${ }^{22-36}$ Barium titanate/nitrile butadiene rubber (BT/NBR) composite, a kind of common piezoelectric composite, has fine piezoelectric property and relatively higher density. This combination of piezoceramics fillers and polymer matrix gives the BT/NBR composite relatively higher airflow resistivity which is beneficial to reflect the sound wave and dissipate the sound energy. Polyurethane (PU) is used widely due to its excellent properties such as stability, elasticity, solvent resistance, and fire resistance. And PU foam, a kind of common foam material, is used to absorb noise usually. In general, the PU foam has good sound absorption property at high frequency, but not at low frequency.

In this paper, two types of sound absorption composites with different stratified structures including double-layer structure and alternating multilayered structure were prepared. PU foam was used as one layer, and BT/NBR composite was used as another layer. The two types of stratified structure composite consisted of double layer or alternating layers of the PU foam and the BT/NBR composite, respectively. The effects of the cell size of the PU foam and the thickness of the PU foam layer on the sound absorption efficiency of the BT/NBR-PU foam composite with double-layer structure were studied. And the effects of the alternating unit number on the sound absorption efficiency of the BT/NBR-PU foam composite with alternating multilayered structure was studied.

\section{Experimental}

\subsection{Materials}

Nitrile rubber (NBR, N41) was produced by Lanzhou petrochemical. Polyurethane (PU) foam was produced by Xingtaili New Materials Factory. Conductive carbon black was provided by Tianjin Ebory Chemical Co. LTD. $\mathrm{BaTiO}_{3}$ powder (BT) was prepared by the authors. Other compounding ingredients were bought locally.

\subsection{Preparation of BT/NBR-PU foam composite}

NBR and the fillers were mixed for $30 \mathrm{~min}$ in the two-roll mill (SK160B, Shanghai Tuolin Rubber Machinery Factory). The formulation of the compound is shown in Table 1 . The BT/NBR composites were placed for $24 \mathrm{~h}$ at ambient temperature and then vulcanized with a plate vulcanization machine for $15 \mathrm{~min}$ at $160{ }^{\circ} \mathrm{C}$ and $15 \mathrm{MPa}$. The BT/NBR composites (the thickness is $1 \mathrm{~mm}$ ) and the $\mathrm{PU}$ foam (the thickness is $25 \mathrm{~mm}$ ) were combined with adhesive to prepare the double-layer structure composites. And the PU foams with different thickness $(10 \mathrm{~mm}$, $20 \mathrm{~mm}, 30 \mathrm{~mm}, 40 \mathrm{~mm}, 50 \mathrm{~mm}$, and $60 \mathrm{~mm}$ ) were also used to prepare the double-layer structure composites. Moreover, the $\mathrm{BT} / \mathrm{NBR}$ composites (the thickness is $1 \mathrm{~mm}$ ) and the PU foam (the thicknesses are $5 \mathrm{~mm}$ and $10 \mathrm{~mm}$, respectively) were combined with adhesive to prepare the alternating multilayered structure composites (as in Fig. 1). The actual shape of the samples with double-layer structure and alternating multilayered structure are shown in Fig. 2.

\subsection{Characterization}

The bubble morphology of the PU foam and the interface morphology of the BT/NBR and the PU foam were observed by using an optical microscope (Nanjing Jiangnan Novel Optics Co. LTD). The sound absorption coefficients of the composites were tested by AWA6128A type standing wave tube (Beijing century JT Technology Development Co. LTD), and the sound absorption coefficients were plotted from 200-2000 Hz.

Table 1 Formulation of the compound

Material

Content (phr)

NBR

100

$\mathrm{ZnO}$

Stearic acid

5

Tetramethyl thiuram disulphide

$N$-Cyclohexyl-2-benzothiazole sulfenamide

$N$-Isopropyl- $N^{\prime}$-phenyl- $p$-phenylene

Conductive carbon black

Carbon black

$\mathrm{BaTiO}_{3}$

Sulfur
1.5

2

1

1

2

18

120

1.5 


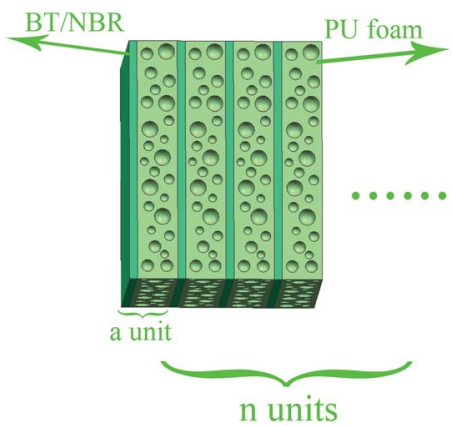

Fig. 1 Schematic of multilayered structure composites.

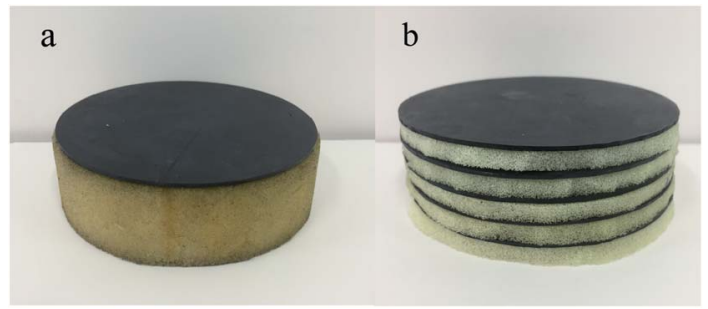

Fig. 2 Actual shape of the BT/NBR-PU foam composites with (a) double-layer structure and (b) alternating multilayered structure.

\section{Results and discussion}

\subsection{Sound absorption performance of the BT/NBR-PU foam composite}

Fig. 3 shows the sound absorption efficiencies of BT/NBR, PU foam, and BT/NBR-PU foam composites. The thickness of BT/
NBR and PU foam is $1 \mathrm{~mm}$ and $25 \mathrm{~mm}$, respectively. And the BT/NBR-PU foam composites were prepared by using the BT/ NBR and the PU foam with the thicknesses above. The PU foam composites have better sound absorption efficiency than the BT/NBR composites, but neither of them has good sound absorption efficiency at low frequency. Actually, the doublelayer structure of the BT/NBR-PU foam composites can enhance the sound absorption efficiency at low frequency. It is worth noting that the variety of the layer facing the sound source greatly affect the sound absorption efficiency of the BT/ NBR-PU foam composites. It can be seen from Fig. 3 that the BT/ NBR-PU foam composite has good sound absorption efficiency at low frequency when the BT/NBR layer faces the sound source. On the contrary, the composite has poor sound absorption efficiency when the PU foam layer faces the sound source. The reason is that the $\mathrm{BT} / \mathrm{NBR}$ layer and the $\mathrm{PU}$ foam layer play different roles in the process of sound absorption. Generally, compared with medium and high frequency sound waves, low frequency sound waves are hard to be absorbed due to its strong penetrability. When the PU foam layer faced the sound source, the medium and high frequency sound waves were absorbed effectively and the low frequency sound waves penetrated the PU foam. However, lots of the sound waves (in especial medium and high frequency sound waves) were reflected in the interface of air and the BT/NBR layer when the BT/NBR layer faced the sound source, because the BT/NBR layer had higher density than the PU foam and had none porous structure. Therefore, most of the low frequency sound waves and a fewer medium and high frequency sound waves entered the interior of the PU foam layer. Thereupon, the porous structure of PU foam was

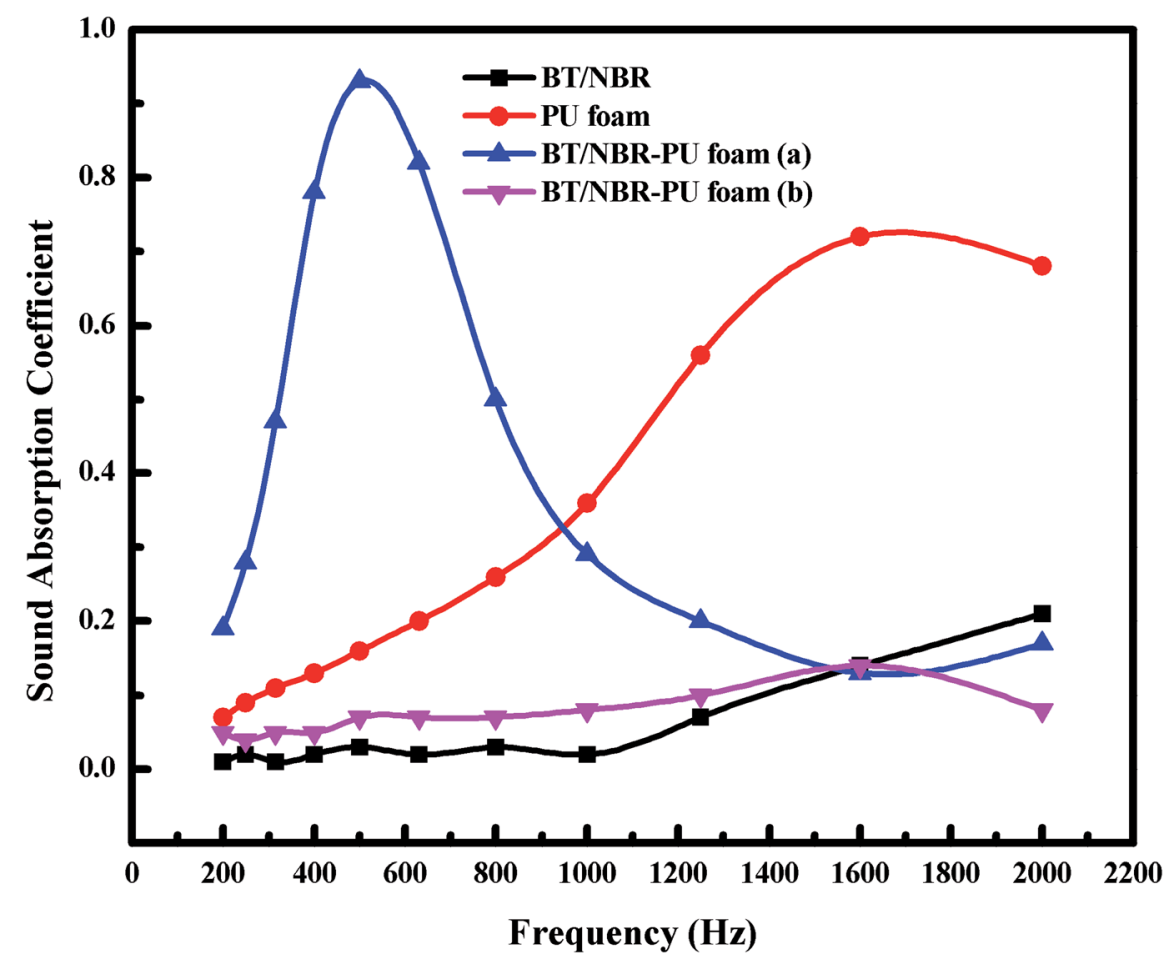

Fig. 3 Sound absorption efficiencies of BT/NBR, PU foam, and BT/NBR-PU foam ((a) BT/NBR layer faced the sound source; (b) PU foam layer faced the sound source) composites. 
applied to absorb the low frequency sound waves rather than the medium and high frequency sound waves. In summary, the $\mathrm{BT} / \mathrm{NBR}$ layer played the role that improved the airflow resistivity and isolated the medium and high frequency sound waves, and the PU foam played the role that absorbed the low frequency sound waves. Moreover, the good absorption at low frequency also benefited from the resonance of the mass per unit area of the nitrile rubber with the combined stiffness of the frame of the PU foam and the air in the holes of the PU foam.

\subsection{Effect of PU foam layer on the sound absorption efficiency}

The acoustic performance of PU foam are related to cell characteristics such as bubble cell size and cell distribution closely, because sound wave can interact with the bubble hole cavity. ${ }^{18}$ And the optical microscope images of PU foam composites were shown in Fig. 4. To unveil the relationship between the microstructure of

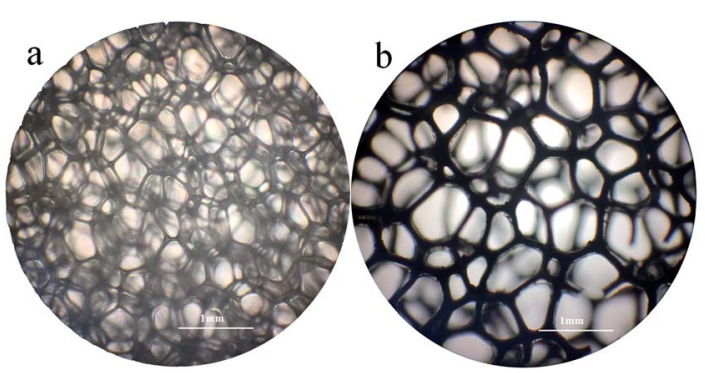

Fig. 4 Optical microscope images of PU foam composites ((a) SCPU; (b) LCPU). the PU foam on the sound absorption property of the BT/NBR-PU foam composites, two types of PU foam with different cell size were used in the composites, respectively, including the PU foam composites with small cells (SCPU composites) and the PU foam composites with large cells (LCPU composites). According to the size distribution in Fig. 5, the distributions of the small cell are more uniform. The average cell size of the SCPU composites is $425.55 \mu \mathrm{m}$, and that of the LCPU composites is $710.00 \mu \mathrm{m}$. The morphologies of the interface of the BT/NBR-SCPU composites and the BT/NBR-LCPU composites are shown in Fig. 6. The interfaces between the BT/NBR layer and PU foam layer are vague, which means that the BT/NBR layer was tightly bonded to the PU foam. Therefore, interface effect and resonance were obvious at the interface between the BT/NBR layer and PU foam layer.

The sound absorption curves of the BT/NBR-SCPU composites and the BT/NBR-LCPU composites have single characteristic peak at low frequency, and both the two composites show excellent sound absorption efficiency at low frequency (as in Fig. 7). The characteristic peak moves toward low frequency with the decrease of cell size. One reason is that the energy causing the air cavity resonance decreased with the decrease of the cell size. Therefore, a low-frequency sound wave with low energy could be absorbed effectively by using the BT/NBR-SCPU composites. Another reason is that the specific surface area of the cell increased with the decrease of cell size. Larger the specific surface area of the cell means longer and more circuitous acoustic wave propagation path, which led to more absorption of the acoustic energy by the composites. In addition, changing the material porosity and pore radius could

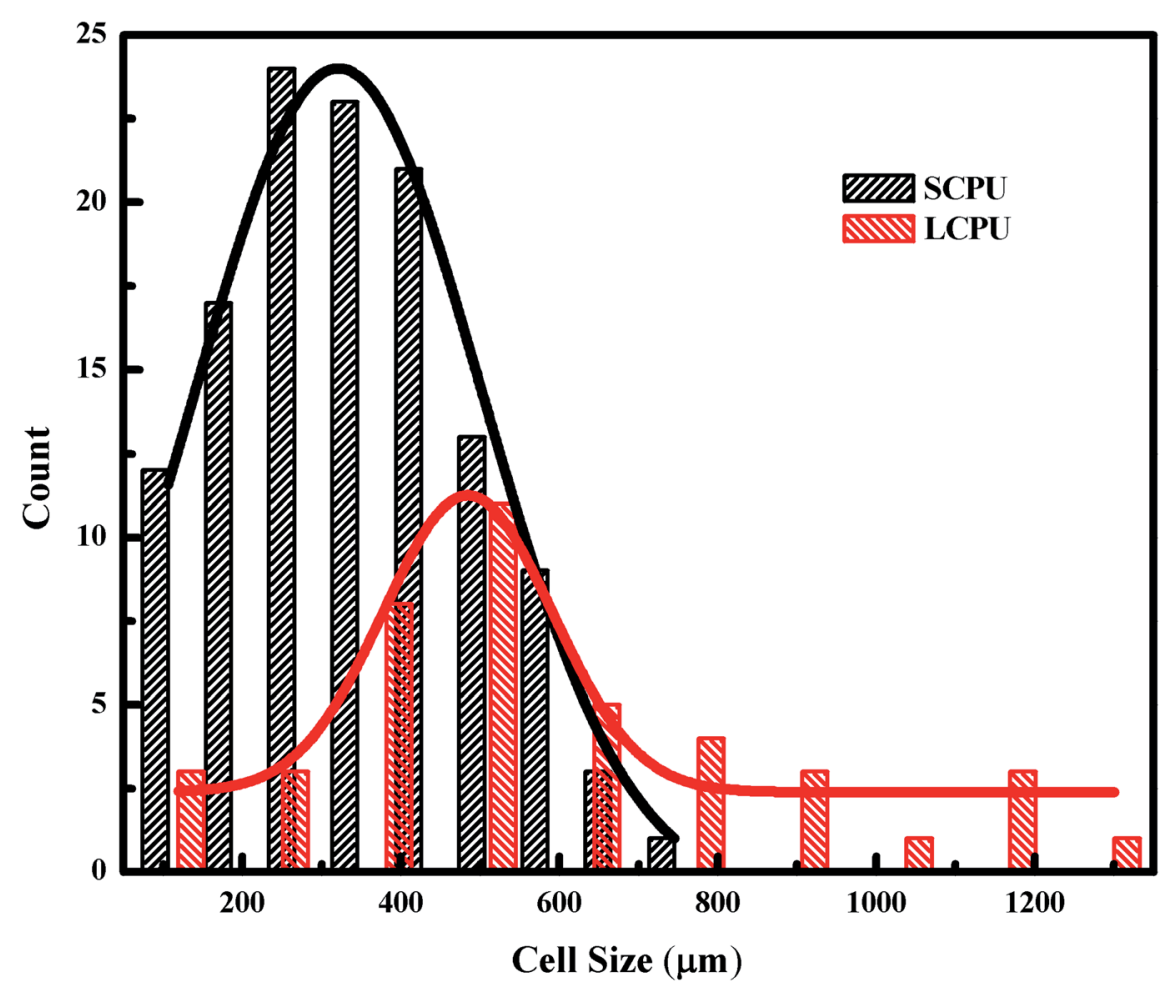

Fig. 5 Cell distributions of PU foam composites. 


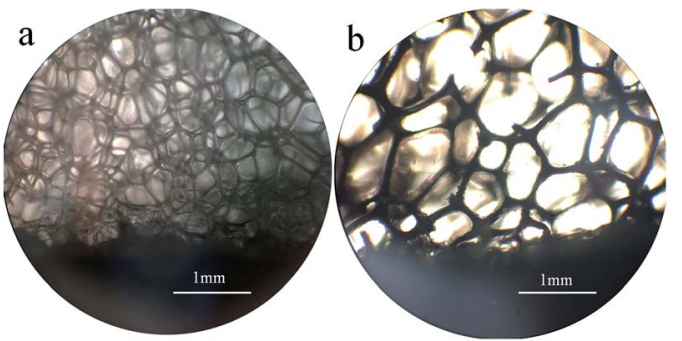

Fig. 6 Optical microscope images of the BT/NBR-PU foam composites ((a) BT/NBR-SCPU; (b) BT/NBR-LCPU).

adjust the flow resistance,$^{37}$ thereby adjusting the sound absorption properties of the composites.

The PU foams with different thickness were also used to prepare the double-layer structure composites to study the effect of the PU foam layer thickness on the sound absorption efficiency of the BT/NBR-PU foam composites (as in Fig. 8). It can be seen that the sound absorption peak of the composites moves toward low frequency with the increase the thickness of PU foam layer. Low frequency sound wave has strong penetrability, and the initial solution is improving the thickness of sound absorbing materials. In general, the composites with large thickness, which have larger acoustic impedance, are easily absorbed the low frequency waves. ${ }^{38}$ The same goes for the BT/NBR-PU foam composites. On the one hand, with the increase of the thickness of the PU foam, the aggregate thickness and the total weight of the BT/NBR-PU foam composite increased. Therefore, the composite has larger acoustic impedance and higher airflow resistivity after increasing the thickness of the PU foam. On the other hand, as mentioned above, the PU foam played the role which absorbed the low frequency sound waves. The increase of the thickness of the PU foam caused the resonant first frequency of the porous layer to move toward the low frequency direction, so the absorption of the low frequency localized sound energy became faster. Furthermore, no matter how the thickness of PU foam changed, it can be found that each BT/NBR-PU foam composites showed excellent sound absorption efficiency in specific frequency range. Therefore, the sound absorption frequency range of the double-layer structure composite could be adjusted by changing the thickness of the PU foam for different application requirements.

\subsection{Effect of alternating multilayered structure on the sound absorption efficiency}

Except the double-layer structure composites, the BT/NBR-PU foam composites with alternating multilayered structure were also prepared to absorb the sound waves. Considering that the aggregate thickness of sound absorption materials should not be too large in practical application, the PU foams with the thickness of $5 \mathrm{~mm}$ and $10 \mathrm{~mm}$ were chosen to prepare the alternating multilayered structure composites. The effect of alternating unit number on the sound absorption efficiency was studied (as in Fig. 9). The results show that the composites have good sound absorption performance with increasing of the alternating unit number in a wider bandwidth of frequency. On the one hand, with the increase of the alternating unit number, more interfaces would arise in the alternating multilayered structure composites, which would bring about more reflection, friction and resonance loss of the sound waves happened on these interfaces. On the other hand, the propagation path of the sound wave would also become longer, because of the more reflection on interfaces and thicker PU foam layer with the increase of the alternating unit number. Accordingly, the

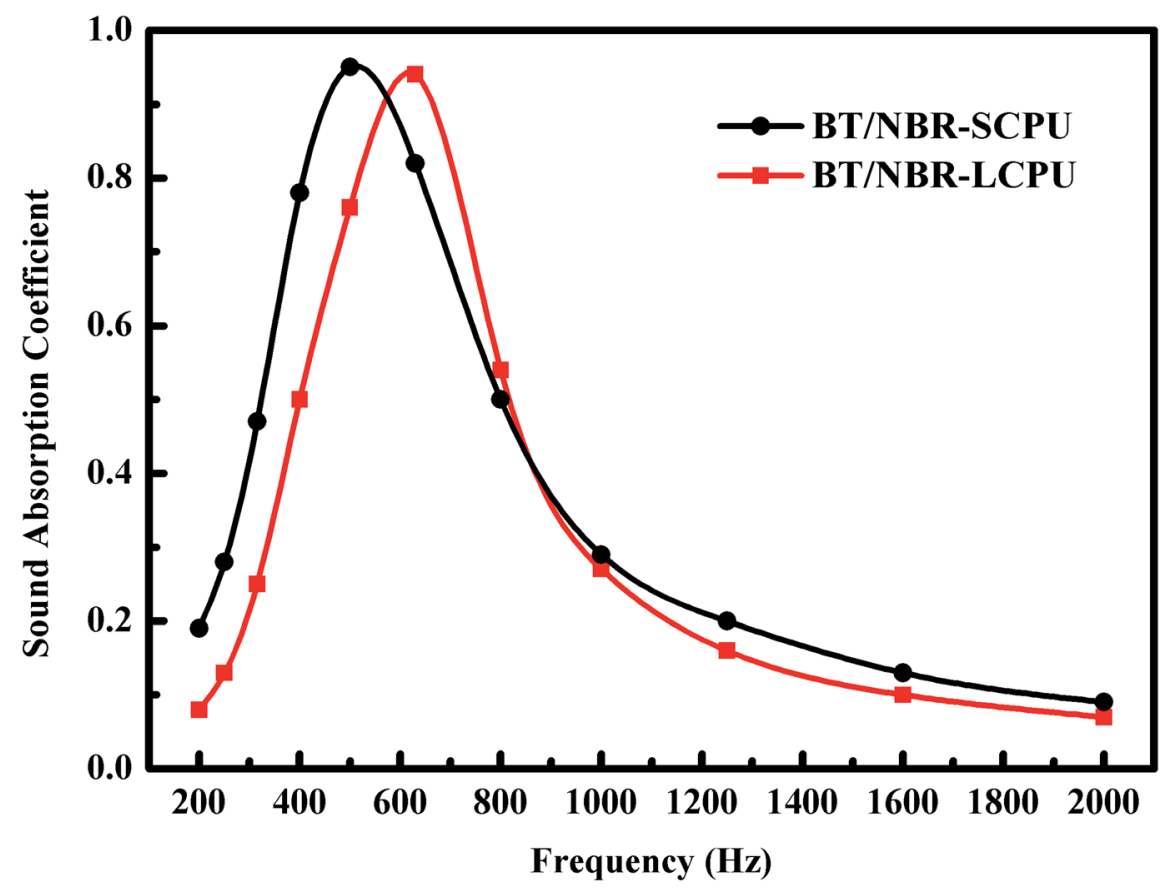

Fig. 7 Sound absorption efficiencies of the BT/NBR-LCPU composites and the BT/NBR-SCPU composites. 


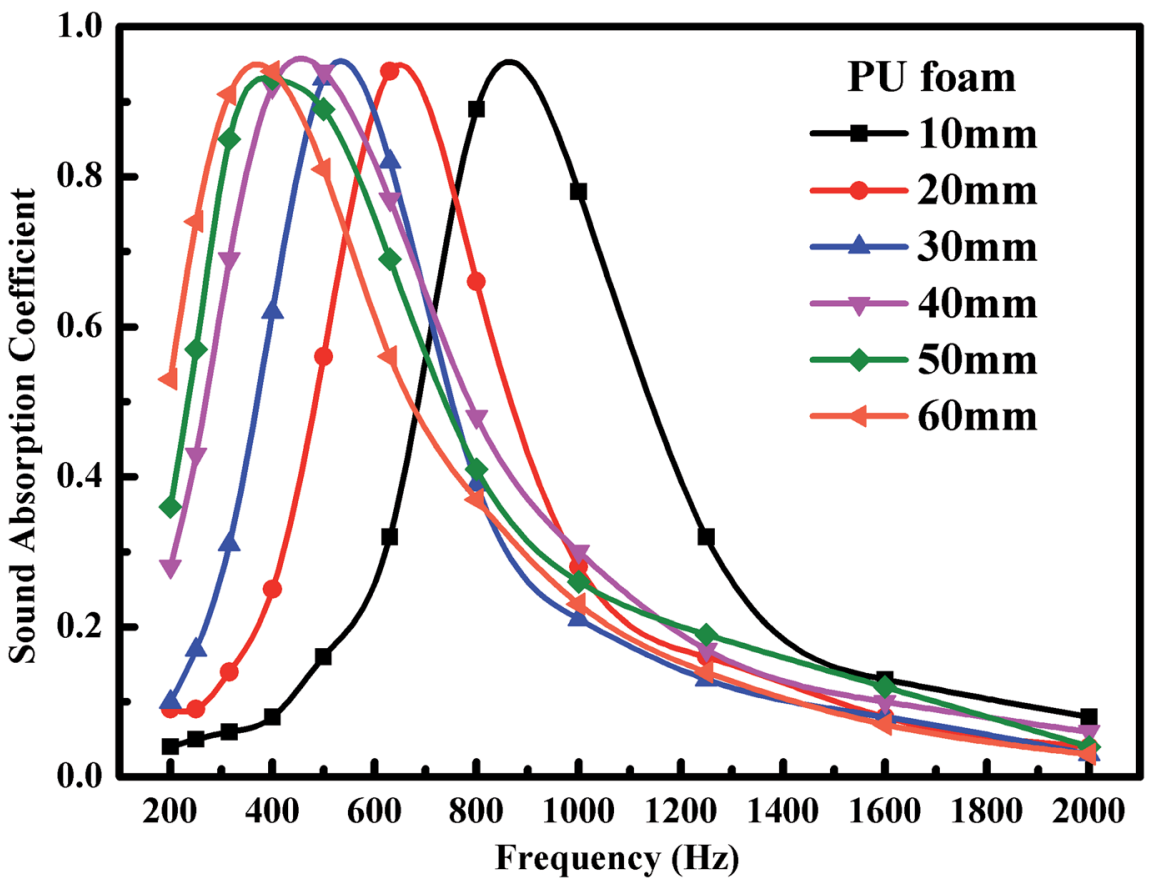

Fig. 8 Effect of the PU foam thickness on sound absorption efficiency of the BT/NBR-PU foam composite.

airflow resistivity of the composites would improve, which was a key non-acoustic parameter determining the sound absorption capability of porous materials. ${ }^{39,40}$ Therefore, the interaction of elasticity matrix, suitable filler, porous structure and alternating multilayered structure leads to the combination of airflow resistivity, resonance absorption, and interface dissipation, which could effectively improve the sound absorption of the material.

Compare with the results reported by previous researchers, it can be concluded that the simply filling such as ramie fiber, ${ }^{7}$ carbon nanotube ${ }^{8}$ piezoelectric ceramic ${ }^{9}$ and other fillers could not greatly improve the low frequency sound absorption performance of composites with polymer matrix. At frequencies below $1000 \mathrm{~Hz}$, the sound absorption coefficients are difficult to reach 0.3. In addition, the structural designs reported before, such as micro-perforated structure, ${ }^{17,18}$ helical structure ${ }^{14}$ and phonon crystal structure, ${ }^{20}$ could have a characteristic peak of sound absorption coefficient above 0.8 at specific frequency. However, the sound absorption frequency band is narrow. The maximum sound absorption coefficient of the foam/film poly(ethylene-co-octene) composite ${ }^{15}$ with alternating multilayer structure can reach about 0.6. Nevertheless, the sound absorption effect is not good enough at frequencies below $1000 \mathrm{~Hz}$. In this study, the average sound absorption coefficient of the BT/NBR-PUSF composites could above 0.5 , and the
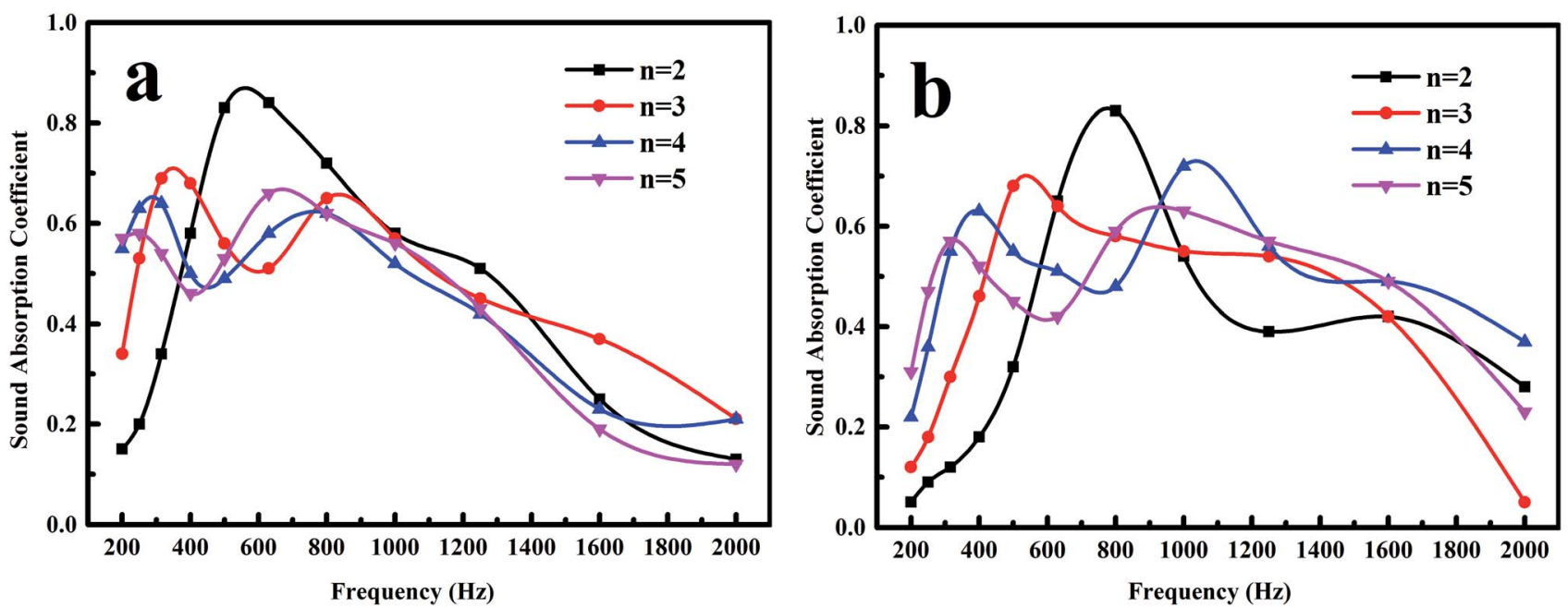

Fig. 9 Effect of alternating unit number on the sound absorption efficiency ((a) the thickness of PU foam was $10 \mathrm{~mm}$; (b) the thickness of PU foam was $5 \mathrm{~mm}$ ). 
maximum sound absorption coefficient could be above 0.9 at a frequency below $800 \mathrm{~Hz}$.

\section{Conclusion}

In this study, the BT/NBR-PU foam composites with two different stratified structures including double-layer structure and alternating multilayered structure were prepared and showed excellent sound absorption performance at low frequency. With the decrease of the cell size of PU foam or with the increase the thickness of PU foam layer, the sound absorption peak of the double-layer structure composites would move toward low frequency. With increasing of the alternating unit number, the composites with alternating multilayered structure have good sound absorption performance in a wider bandwidth of frequency. The alternating multilayered structure could improve the sound absorption efficiency due to more interface reflection and friction loss. Meanwhile, the increase of the thickness also increases the airflow resistivity of the composite, resulting in better low frequency absorption. The BT/NBR-PU foam composites (the thickness of PU foam is 5 $\mathrm{mm}$, and $n$ equals 4 or 5 ) showed best sound absorption efficiency in wider bandwidth of frequency. Through intelligent design of cell size, layer thickness and alternating unit number of PU foam, the sound absorption range can be adjusted for different application requirements and the optimal sound absorption effect can be achieved. Therefore, the stratified structure is a feasible approach in order to improve acoustic efficiency of sound absorption materials used in construction, transportation and other fields.

\section{Conflicts of interest}

There are no conflicts to declare.

\section{Acknowledgements}

This work was supported by the National Natural Science Foundation of China (51273154), the Natural Science Foundation of Hubei (2011CBD220) and the Research Project of Hubei Provincial Department of Education (D20171505).

\section{References}

1 Y. Wang, C. Zhang, L. Ren, M. Lchchou, M. Galland and O. Bareille, Polym. Compos., 2013, 34, 1847-1855.

2 F. Chevillotte, Appl. Acoust., 2012, 73, 56-60.

3 T. C. Hung, J. S. Huang, Y. W. Wang and K. Y. Lin, Constr. Build. Mater., 2014, 50, 328-334.

4 M. A. Pereira and N. C. Branco, Prog. Biochem. Biophys., 2007, 93, 256-279.

5 A. Kaczmarska and A. Luczak, Prog. Biochem. Biophys., 2007, 13, 117-125.

6 C. Baliatsas, K. L. Van, P. R. Van and J. Yzermans, Sci. Total Environ., 2016, 163, 557-558.

7 D. Chen, J. Li and J. Ren, Composites, Part A, 2010, 41, 10121018.
8 M. W. Chang and H. C. Min, Compos. Sci. Technol., 2016, 127, 127-133.

9 A. Kundu and A. Berry, J. Intell. Mater. Syst. Struct., 2011, 22, 1771-1787.

10 Y. Wu, X. Sun, W. Wu, X. Lin, X. Shen, Z. Wang, R. K. Y. Li, Z. Yang, K. T. Lau and J. K. Kim, Composites, Part A, 2017, 102, 391-399.

11 W. Zhai, X. Yu, X. Song, L. Y. L. Ang, F. Cui, H. P. Lee and T. Li, Mater. Des., 2017, 137, 108-116.

12 P. Wang, L. Lu and K. Bertoldi, Phys. Rev. Lett., 2015, 115, 104302.

13 G. Wang, X. Wen, J. Wen, L. Shao and Y. Liu, Phys. Rev. Lett., 2004, 93, 154302.

14 S. Babaee, N. Viard, P. Wang, N. X. Fang and K. Bertoldi, Adv. Mater., 2016, 28, 1631-1635.

15 T. Zhao, M. Yang, H. Wu, S. Guo, X. Sun and W. Liang, Mater. Lett., 2015, 139, 275-278.

16 H. Meng, M. A. Galland, M. Lchchou, O. Bareille, F. X. Xin and T. J. Lu, Compos. Struct., 2017, 182, 1-11.

17 P. Leclaire, O. Umnova, T. Dipont and R. Panneton, J. Acoust. Soc. Am., 2015, 137, 1772-1782.

18 N. M. Baouche, H. D. Baynast, A. Lebert, S. Sun, P. Leclaire and P. Michaud, Ind. Crops Prod., 2014, 58, 244-250.

19 X. H. Yang, S. W. Ren, W. B. Wang, X. Liu, F. X. Xin and T.J Lu, Compos. Sci. Technol., 2015, 118, 276-283.

20 Z. Y. Liu, X. X. Zhang, Y. W. Mao, Y. Y. Zhu, Z. Y. Yang, C. T. Chan and P. Sheng, Science, 2000, 289, 1734-1736.

21 B. Xue, R. Li, J. G. Deng and J. H. Zhang, Ind. Eng. Chem. Res., 2016, 55, 3982-3989.

22 X. L. Jiang, Z. Yang, Z. J. Wang, F. Q. Zhang, F. You and C. Yao, Materials, 2018, 11, 474-480.

23 T. Lu, H. Pan, J. Ma, Y. Li, S. W. Bokhari, X. L. Jiang, S. Zhu and D. Cellulose, Mater. Interfaces, 2017, 9, 18231-18237.

24 X. L. Jiang, C. J. Li, S. Liu, F. Q. Zhang, F. You and C. Yao, RSC Adv., 2017, 7, 24598-24606.

25 R. Chen, X. L. Jiang, F. You and C. Yao, Fibers Polym., 2016, 17, 1550-1557.

26 X. L. Jiang, X. Xu, T. Geng, F. You, W. Wang and C. Yao, Polymer, 2016, 40, 684-690.

27 X. L. Jiang, J. Zhang, L. Yu and F. You, Chin. J. Inorg. Chem., 2016, 32, 1337-1344.

28 X. L. Jiang, L. Yu, C. Yao, F. You and J. Zhang, Nano, 2016, 11, 67-74.

29 X. L. Jiang, L. Yu, F. Q. Zhang and J. Zhang, Materials, 2016, 9, 323-333.

30 X. L. Jiang, J. Zhang, L. Yu and R. Chen, Micro Nano Lett., 2016, 11, 137-141.

31 X. L. Jiang, W. Wang, J. Guan, H. Yang, G. Sun and J. Ren, Rare Met. Mater. Eng., 2015, 44, 1249-1253.

32 X. L. Jiang, Y. Du, W. Wang, H. Yang and J. Ren, Rare Met. Mater. Eng., 2014, 43, 249-252.

33 X. L. Jiang, Y. Fan and F. Li, J. Thermoplast. Compos. Mater., 2013, 26, 1192-1205.

34 X. Lou, C. Zhu, H. Pan, J. Ma, S. Zhu, D. Zhang and X. Jiang, Electrochim. Acta, 2016, 205, 70-76.

35 X. L. Jiang and Y. Fan, J. Appl. Polym. Sci., 2012, 124, 24232429. 
36 Y. Sun, Z. Fang, C. Wang, A. Zhou and H. Duan, 39 N. Sebaa, Z. E. A. Fellah, M. Fellah, W. Lauriks and Nanotechnology, 2015, 26, 374002.

C. Depollier, J. Appl. Phys., 2005, 98, 084901.

37 S. B. Park and M. Tia, Cem. Concr. Res., 2004, 34, 177-184. 40 Z. E. A. Fellah, M. Fellah, N. Sebaa, W. Lauriks and 38 O. Buser, J. Sound Vib., 1986, 111, 71-92. C. Depollier, J. Acoust. Soc. Am., 2006, 119, 1926-1928. 